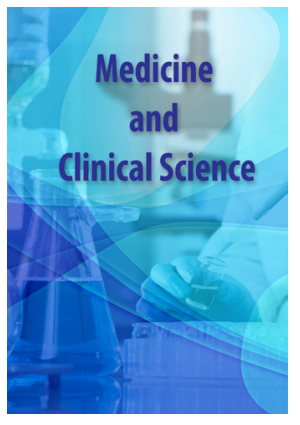

Correspondence

Akiko Noda, PhD

Chubu University Graduate School of Life and Health Sciences, 1200 Matsumoto-cho, Kasugai-shi, Aichi Pref. 487-8501, Japan

Tel: +81-568-51-9906

Fax +81-568-51-5370

E-mail: anoda@isc.chubu.ac.jp

- Received Date: 31 Dec 2019;

- Accepted Date: 07 Jan 2020;

- Publication Date: 14 Jan 2020

Keywords

Obese, Hypertension, Cardiovascular Events, LV Mass, Sleep
Copyright

(c) 2020 Science Excel. This is an open access article distributed under the terms of the Creative Commons Attribution 4.0 International license.

\title{
False-negative electrocardiographic left ventricular hypertrophy in patients with obstructive sleep apnea: Significances of echocardiography and 24-hour blood pressure
}

\author{
Akiko Noda' ${ }^{1}$ Seiko Miyata ${ }^{2}$, Fumihiko Yasuma ${ }^{3}$ and Yoshinari Yasuda ${ }^{4}$ \\ ${ }^{1}$ Chubu University Graduate School of Life and Health Sciences, Kasugai, Japan \\ ${ }^{2}$ Department of Psychiatry, Nagoya University Graduate School of Medicine, Nagoya, Japan \\ ${ }^{3}$ Department of Internal Medicine, Suzuka National Hospital, Suzuka, Japan \\ ${ }^{4}$ Department of CKD Initiatives, Nagoya University Graduate School of Medicine, Nagoya, Japan
}

\begin{abstract}
Early recognition of left ventricular hypertrophy (LVH) in patients with obstructive sleep apnea (OSA) is an important clinical issue in sleep and cardiovascular medicine. Obesity may influence discrepancies in electrocardiographic and echocardiographic LVH detection in patients with OSA Electrocardiography (ECG), echocardiography (Echo), and 24-hour ambulatory blood pressure monitoring were performed in 80 consecutive patients with OSA and 32 age-matched controls. Patients with OSA were classified into the following groups: group 1: ECG-LVH(+) and Echo-LVH(+); group 2 (false-negative ECG-LVH): ECG-LVH(-) and Echo-LVH(+); group 3(false-positive ECG-LVH): ECG-LVH(+) and Echo-LVH(-); and group 4: ECG-LVH(-) and Echo-LVH(-).The prevalence of ECG-LVH and Echo-LVH was $27.5 \%(22 / 80)$ and $37.5 \%(30 / 80)$, respectively. False-negative ECG-LVH results (group 2) were obtained in $50.0 \%$ (15/30) of patients. Overall, there was no correlation between QRS voltage and LV mass/body surface area. The ECG axis was significantly deviated to the left in groups 1,2 and 4 compared to the controls. RaVL and SV3+RaVL were higher in group 2 than the controls. Although the ECG axis and body mass index were similar in groups 1 and 2, LV mass/body surface area was significantly greater in group 1 than group 2. Mean 24-hour systolic and diastolic blood pressures were significantly elevated in groups 1 and 2 than groups 3 and 4. Obesity in patients with OSA may play an important role regarding differences in ECG amplitudes. Considering the high prevalence of false-negative ECG-LVH, evaluation of LVH using Echo is necessary to screen the risk of cardiovascular events in obese and hypertensive patients with OSA.
\end{abstract}

\section{Introduction}

Obstructive sleep apnea (OSA) is characterized by recurrent episodes of apnea, hypopnea, hypoxia, arousal, and sleep fragmentation, and also it is known to have various cardiovascular complications [1-3]. Prospective populationbased epidemiological studies have shown that individuals with OSA are two to three times more likely to develop hypertension than those without OSA [4,5]. A high apnea/hypopnea index (AHI) or a long period of sleep with oxygen saturation below $90 \%$ has a dose-response relationship with hypertension after adjusting the primary potential confounders (e.g., age, sex, BMI, alcohol intake, and smoking) [6]. We previously reported that left ventricular hypertrophy $(\mathrm{LVH})$ in patients with severe OSA was more prevalent than those with mild OSA, suggesting that frequent episodes of oxygen desaturation and/or arousal during sleep may precipitate the long-term elevation in blood pressure (BP) and LVH [7]. Thus, early recognition of LVH in OSA is an important clinical issue in sleep and cardiovascular medicine.

$\mathrm{LVH}$ is a complication of hypertension that predicts a higher incidence of cardiovascular disease and death due to stroke, heart attack, and other atherosclerotic diseases [8]. Electrocardiography (ECG) is an inexpensive, noninvasive, and convenient method for screening LVH in large populations [9]. However, the accuracy and predictability of ECG as a diagnostic tool is practically tentative due to frequency of false-positive and false-negative findings, particularly in patients with OSA. From the technical view point, ECG for detecting LVH cannot always be reliable due to such extracardiac factors as sex, age, weight, and chest-wall configuration $[9,10]$. We hypothesized that obesity might influence discrepancies in detecting LVH with ECG and ecocardiography (Echo) in patients with OSA.

In this study, we examined the relationship between ECG-LVH, Echo-LVH, and 24-hour BP in patients with OSA.

\section{Methods}

\section{Subjects}

A total of 80 consecutive male patients with OSA (mean \pm SD, $58.1 \pm 7.6$ years; range, $50-68$ ) and 32 agematched healthy males ( $45.5 \pm 3.6$ years; range, $40-60)$ without OSA were included in the study. Subjects in the control group without cardiovascular diseases 
or diabetes mellitus did not take any medications for other systemic complications. Exclusion criteria for the study were complete bundle branch block and Wolff-Parkinson-White syndrome in ECG, left ventricular dilation, right ventricular hypertrophy, and abnormal wall motion in Echo and histories of ischemic heart disease, cerebrovascular accidents, and current electrolyte imbalance. All of the above factors are known to interfere with detection of LVH [11]. The study protocol was approved by an institutional review committee. All subjects were informed of the experimental protocol, and written informed consent was obtained.

\section{Electrocardiographic data}

Twelve-lead ECGs were recorded and LVH was defined according to the voltage criteria of Sokolow and Lyon (RV5 or RV6 $\geq 2.6 \mathrm{mV}$; SV1+RV5 and/or SV1+RV6 $\geq 3.5 \mathrm{mV}$ ) [12]. The ECG axis, RV1-6, SV1-6, SV1+RV5, $\mathrm{SV}_{1}+\mathrm{RV} 6, \mathrm{RaVL}$, and SV3+RaVL were measured.

\section{Echocardiographic data}

Long-axis, short-axis, and apical two-chamber and four-chamber views of the two dimensional Echo were obtained for each subject. Enddiastolic left ventricular internal dimension (LVDd), left ventricular posterior wall thickness (LVPWT), and interventricular septal thickness (IVST) were measured using the Penn convention [13]. LV mass/body surface area (LV mass index) was estimated according to Devereux et al. [11,13].

\section{4-hour ambulatory blood pressure monitoring}

After attaching an appropriately sized cuff to the patient's non-dominant arm, 24-hour ambulatory BP was measured continuously at 30-minute intervals using an oscillometric method (TM-2431, A\&D Co. Inc., Tokyo, Japan). Subjects were allowed to perform routine daily activities during BP monitoring, and a diary was provided to record subjective symptoms or changes in physical or mental conditions. Artifact data were identified by experienced biomedical laboratory scientists and omitted from analyses. Hypertension based on 24-hour ambulatory BP was defined when the mean daytime systolic $\mathrm{BP}$ (SBP) was equal to or greater than $130 \mathrm{mmHg}$ or the mean daytime diastolic BP (DBP) was equal to or greater than $80 \mathrm{mmHg}$ according to established guidelines [14].

\section{Polysomnographic data}

Electroencephalograms and electrooculograms were continuously recorded during polysomnography. Respiration was monitored using an oronasal thermistor and thoracoabdominal strain gauge. Apnea was defined as a cessation of airflow through the mouth and nose for 10 $\mathrm{s}$, and hypopnea was defined as a reduction in airflow associated with either an oxygen desaturation of $>3 \%$ or arousal, also for $\geq 10 \mathrm{~s}$ [15]. The apnea-hypopnea index (AHI) was determined as the number of apnea and hypopnea episodes per hour. Individuals with an $\mathrm{AHI}$ of $\geq 5 / \mathrm{h}$ and daytime sleepiness were diagnosed as OSA. The time during which the nocturnal oxygen saturation $\left(\mathrm{SpO}_{2}\right)$ was $<90 \%$ (oxygen desaturation time, or ODT) was measured with a pulse oximeter.

\section{Statistical analysis}

Patients with OSA were classified into the following groups: group 1 : ECG-LVH(+) and Echo-LVH(+); group 2 (false-negative ECG-LVH): ECG-LVH(-) and Echo-LVH(+); group 3 (false-positive ECG-LVH): ECG-LVH(+) and Echo-LVH(-); and group 4: ECG-LVH(-) and Echo-LVH(-). Values are expressed as mean \pm SD. Data were analyzed with Statview (SAS Institute Inc., Cary, NC) using analysis of variance (ANOVA) with Scheffe's test for multiple comparisons. Correlations between ECG indices (RV1-6, SV1-6, SV1+RV5, SV1+RV6, RaVL, and $\mathrm{SV}_{3}+\mathrm{RaVL}$ ) and Echo indices (IVST, LVPWT, (IVST + LVPWT)/2, and LV mass index) were assessed by linear regression. $P<0.05$ was considered statistically significant.

\section{Results}

Subject characteristics are shown in Table 1. The overall prevalence of ECG-LVH and Echo-LVH was $27.5 \%(22 / 80)$ and $37.5 \%(30 / 80)$, respectively. The proportion of subjects in groups $1,2,3$, and 4 were $18.8 \%, 18.8 \%, 8.8 \%$, and $53.4 \%$, respectively. The overall ratio of the discrepancy between ECG-LVH and Echo-LVH detection was 27.5\% $(22 / 80)$. False-negative ECG-LVH results (group 2) were obtained in $50.0 \%(15 / 30)$.

There were no significant differences among the four groups regarding AHI. The oxygen desaturation time was significantly longer in group 2 than groups 3 and 4 . Mean 24-hour SBP and DBP were significantly higher in groups 1 and 2 than groups 3 and 4 . Body mass index (BMI) was significantly greater in group 1 than group 4 (Table 1 ).

The ECG axis significantly deviated to the left in groups 1,2 , and 4 compared to the controls. RV1 was significantly lower and SV5 was significantly higher in group 2 compared to the controls. $\mathrm{SV}_{3}, \mathrm{SV}_{4}$, and SV5 were significantly higher in group 2 than group 4 . RaVL and SV3+ $\mathrm{RaVL}$ were higher in group 2 compared to the controls (Table 2).

There was no correlation between QRS voltage and LV mass index in total group, groups 1, 2, 3, and 4 and subjects with Echo-LVH (+).

\begin{tabular}{|l|c|c|c|c|}
\hline & $\begin{array}{c}\text { Group 1 } \\
\text { ECG-LVH(+) } \\
\text { Echo-LVH(+) }\end{array}$ & $\begin{array}{c}\text { Group 2 } \\
\text { ECG-LVH(-) } \\
\text { Echo-LVH(+) }\end{array}$ & $\begin{array}{c}\text { Group 3 } \\
\text { ECG-LVH(+) } \\
\text { Echo-LVH(-) }\end{array}$ & $\begin{array}{c}\text { Group 4 } \\
\text { ECG-LVH(-) } \\
\text { Echo-LVH(-) }\end{array}$ \\
\hline $\mathrm{n}$ & 15 & 15 & 7 & 43 \\
\hline Age (years) & $57.4 \pm 10.1$ & $58.6 \pm 9.52$ & $60.9 \pm 6.69$ & $56.1 \pm 8.38$ \\
\hline Weight $(\mathrm{kg})$ & $80.2 \pm 15.6$ & $71.4 \pm 11.3$ & $70.0 \pm 10.6$ & $71.1 \pm 10.5^{*}$ \\
\hline Height $(\mathrm{cm})$ & $168 \pm 6.55$ & $164 \pm 6.65$ & $166 \pm 6.32$ & $166 \pm 4.74$ \\
\hline BMI $\left(\mathrm{kg} / \mathrm{m}^{2}\right)$ & $28.2 \pm 4.22$ & $26.7 \pm 4.61$ & $25.4 \pm 2.65$ & $25.7 \pm 3.44^{*}$ \\
\hline AHI $(/ \mathrm{h})$ & $32.5 \pm 17.9$ & $36.9 \pm 22.1$ & $20.6 \pm 7.60$ & $28.6 \pm 17.7$ \\
\hline ODT $(\mathrm{min})$ & $83.1 \pm 76.9$ & $129 \pm 129$ & $37.9 \pm 36.5^{\#}$ & $52.8 \pm 61.6^{\#}$ \\
\hline 24-hour meanSBP $(\mathrm{mmHg})$ & $148 \pm 10.4$ & $146 \pm 9.80$ & $130 \pm 15.0^{* \#}$ & $134 \pm 8.43^{* \#}$ \\
\hline 24-hour meanDBP $(\mathrm{mmHg})$ & $94.4 \pm 6.63$ & $91.1 \pm 9.48$ & $83.3 \pm 5.66^{* *}$ & $84.4 \pm 6.57^{* \#}$ \\
\hline
\end{tabular}

$*: \mathrm{p}<0.05$ vs group 1, \#: $\mathrm{p}<0.05$ vs group 2. ECG, electrocardiography, LVH, left ventricular hypertrophy; Echo, echocardiography;

BMI, body mass index; AHI, apnea/hypopnea index; ODT, oxygen desaturation time; SBP, systolic blood pressure; DBP, diastolic blood pressure

Table 1. Characteristics of subjects 


\begin{tabular}{|l|c|c|c|c|c|}
\hline & Controls & $\begin{array}{c}\text { Group 1 } \\
\text { ECG-LVH(+) } \\
\text { Echo-LVH(+) }\end{array}$ & $\begin{array}{c}\text { Group 2 } \\
\text { ECG-LVH(-) } \\
\text { Echo-LVH(+) }\end{array}$ & $\begin{array}{c}\text { Group 3 } \\
\text { ECG-LVH(+) } \\
\text { Echo-LVH(-) }\end{array}$ & $\begin{array}{c}\text { Group 4 } \\
\text { ECG-LVH(-) } \\
\text { Echo-LVH(-) }\end{array}$ \\
\hline QRS axis & $52.7 \pm 22.6$ & $29.7 \pm 27.4^{*}$ & $29.9 \pm 48.2^{*}$ & $47.3 \pm 32.0$ & $32.9 \pm 27.1^{*}$ \\
\hline RV1 & $0.356 \pm 0.226$ & $0.345 \pm 0.250$ & $0.162 \pm 0.161^{*}$ & $0.358 \pm 0.124^{\#}$ & $0.273 \pm 0.188$ \\
\hline RV2 & $0.748 \pm 0.304$ & $0.850 \pm 0.606$ & $0.538 \pm 0.336$ & $0.783 \pm 0.223$ & $0.669 \pm 0.413$ \\
\hline RV3 & $0.928 \pm 0.276$ & $1.31 \pm 1.08$ & $1.00 \pm 0.492$ & $1.07 \pm 0.236$ & $1.14 \pm 0.641$ \\
\hline RV4 & $1.59 \pm 0.474$ & $2.27 \pm 1.60$ & $1.73 \pm 0.450$ & $1.77 \pm 0.485$ & $1.71 \pm 0.651$ \\
\hline RV5 & $1.73 \pm 0.440$ & $3.00 \pm 1.26^{*}$ & $1.87 \pm 0.327^{\#}$ & $2.52 \pm 0.262^{* \dagger}$ & $1.75 \pm 0.453^{\# \ddagger}$ \\
\hline RV6 & $128 \pm 0.383$ & $1.91 \pm 0.716^{*}$ & $1.22 \pm 0.298^{\#}$ & $1.89 \pm 0.199^{* \dagger}$ & $1.27 \pm 0.311^{\# \ddagger}$ \\
\hline SV1 & $1.14 \pm 0.553$ & $1.30 \pm 0.751$ & $0.969 \pm 0.421$ & $1.13 \pm 0.151$ & $0.860 \pm 0.340^{* \#}$ \\
\hline SV2 & $1.87 \pm 0.713$ & $1.56 \pm 0.789$ & $1.71 \pm 0.485$ & $1.73 \pm 0.360$ & $1.34 \pm 0.424^{*}$ \\
\hline SV3 & $1.47 \pm 0.539$ & $1.41 \pm 0.917$ & $1.82 \pm 0.581$ & $1.59 \pm 0.450$ & $1.14 \pm 0.487^{* \dagger}$ \\
\hline SV4 & $1.02 \pm 0.910$ & $1.00 \pm 0.522$ & $1.39 \pm 0.558$ & $1.09 \pm 0.552$ & $0.785 \pm 0.418^{\dagger}$ \\
\hline SV5 & $0.452 \pm 0.449$ & $0.571 \pm 0.415$ & $0.758 \pm 0.428^{*}$ & $0.508 \pm 0.350$ & $0.387 \pm 0.249^{\dagger}$ \\
\hline SV6 & $0.153 \pm 0.272$ & $0.211 \pm 0.202$ & $0.237 \pm 0.217$ & $0.167 \pm 0.140$ & $0.144 \pm 0.141$ \\
\hline SV1+RV5 & $2.87 \pm 0.734$ & $4.30 \pm 1.72^{*}$ & $2.84 \pm 0.510^{\#}$ & $3.65 \pm 0.237^{* \dagger}$ & $2.61 \pm 0.498^{\# \ddagger}$ \\
\hline SV1+RV6 & $2.42 \pm 0.709$ & $3.21 \pm 1.32^{*}$ & $2.10 \pm 0.629^{\#}$ & $3.03 \pm 0.216^{\dagger}$ & $2.13 \pm 0.421^{\# \ddagger}$ \\
\hline RaVL & $0.264 \pm 0.220$ & $0.711 \pm 0.394^{*}$ & $0.508 \pm 0.350^{*}$ & $0.455 \pm 0.493$ & $0.431 \pm 0.270^{* \#}$ \\
\hline RaVL+SV3 & $1.73 \pm 0.520$ & $2.12 \pm 1.11$ & $2.30 \pm 0.614^{*}$ & $2.05 \pm 0.644$ & $1.57 \pm 0.518^{* \dagger}$ \\
\hline
\end{tabular}

*: $\mathrm{p}<0.05$ vs controls, \#: $\mathrm{p}<0.05$ vs group $1, \uparrow: \mathrm{p}<0.05$ vs group $2, \neq: \mathrm{p}<0.05$ vs group 3 . ECG, electrocardiography; LVH, left ventricle hypertrophy; Echo, echocardiography.

Table 2. Comparison of ECG findings among controls and patients with OSA

\section{Discussion}

We found the high prevalence of false-negative ECG-LVH (50.0\%). The severity of OSA and BP were significantly higher in the ECG$\mathrm{LVH}(-) / \mathrm{Echo}-\mathrm{LVH}(+)$ group than the Echo-LVH(-) group. BMI was significantly greater in the ECG-LVH(-)/Echo-LVH(+) group than ECG-LVH(-)/Echo-LVH(-) group. These findings indicate that LVH detection using both 12-lead ECG and Echo is mandatory especially in obese and hypertensive patients with OSA.

Obesity is considered a cause left axis shift of ECG, which is associated with an upward shift of the diaphragm and a horizontal shift of heart at the anatomy. Increased subcutaneous fat in the upper body as a result of obesity can attenuate ECG amplitudes in the left lateral leads, such as RV5 and increased $\mathrm{RaVL}$, due to elevated impedance in fat tissue and increased distance from the cardiac electrical source to recording sites [10]. In the present study, there was a significant left axis deviation in ECG, and the voltage of $\mathrm{RaVL}$ and $\mathrm{RaVL}+\mathrm{SV} 3$ were significantly greater in patients with OSA in the ECG-LVH(-)/Echo-LVH(+) group than the controls.

The relationship between voltage on body surface ECG $(\mathrm{V})$ and cardiac electromotive force $(\mathrm{H})$ is represented as $\mathrm{V}=$ lead vector $\times \mathrm{H}$ [1618]. The lead vector is based on body fluid electric ratio, position of the heart and parapet, anisotropic lung tissue around the heart, and distance from the exploring electrode to the center of the heart [19]. The ECG axis and BMI were similar in the ECG-LVH $(+) /$ Echo-LVH $(+)$ and ECG-LVH(-)/Echo-LVH(+) groups. Therefore, the lead vector compared to electromotive force in the ECG-LVH(-)/Echo-LVH(+) group may be relatively greater than the ECG-LVH(+)/Echo-LVH(+) group, which could result in false-negative ECG-LVH results.

There was no correlation between QRS voltage and LV mass index in all of the patients and patients with Echo-LVH(+). Generally, ECG has a relatively poor sensitivity for $\mathrm{LVH}$, and there is a poor correlation between the criterion of Sokolow and Lyon and LV mass [20,21]. However, we showed a significant correlation between QRS voltage and LV mass in subjects with Echo-LVH(+), indicating that the lead vector influences body surface ECG [22]. Relationships between the lead vector and electromotive force could result in differences between groups with respect to correlation. Therefore, increased body weight in patients with OSA may play a significant role regarding differences in ECG amplitudes in subjects with and without Echo-LVH(+).

Hypertension as a complication of OSA is attributed to a poor prognosis [23]. Continuous positive airway pressure therapy for OSA reduces the risk of cardiovascular complications secondary to endothelial dysfunction or increased sympathetic activity [24]. Our recent study showed that bilevel positive airway pressure therapy was effective for treating not only severe OSA but also sleep quality and autonomic activity [25]. LVH with diastolic left ventricular dysfunction is a major indicator of cardiovascular disease $[8,26]$, and it predicts a higher incidence of clinical events attributable to cardiovascular diseases and death $[8,27]$. Therefore, evaluation of LVH using Echo is necessary for early diagnosis and treatment of LVH in patients with OSA and/or hypertension, particularly in obese patients.

\section{Conclusions}

For cases in which ECG does not detect LVH, 24-hour ambulatory BP monitoring and Echo may be useful tools for preventing cardiac events in patients with OSA.

\section{Conflict of interest}

The authors declare that they have no conflict of interest.

\section{References}

1. Phillipson EA. Sleep apnea-A major public health problem. N Engl J Med. 1993;328:1271-1273.

2. Svanborg E, Larsson H. Development of nocturnal respiratory disturbance in untreated patients with obstructive sleep apnea syndrome. Chest. 1993;104:340-343.

3. Peppard PE, Young T, Palta M, Skatrud J. Prospective Study of the 
Association between sleep-Disordered Breathing and Hypertension. New England Journal of Medicine. 2000;342:1378-1384.

4. O'Connor GT, Caffo B, Newman AB, et al. Prospective Study of Sleepdisordered Breathing and Hypertension: The Sleep Heart Health Study. Am J Respir Crit Care Med. 2009;179:1159-1164.

5. Nieto FJ, Young TB, Lind BK, et al. Association of Sleep-Disordered Breathing, Sleep Apnea, and Hypertension in a Large CommunityBased Study. JAMA. 2000;283:1829-1836.

6. Noda A, Okada T, Hayashi H, et al. 24-hour ambulatory blood pressure variability in obstructive sleep apnea syndrome. Chest. 1993;103:13431347.

7. Noda A, Okada T, Yasuma F, et al. Cardiac hypertrophy in obstructive sleep apnea syndrome. Chest. 1995;107:1538-1544.

8. Levy D, Garrison RJ, Savage DD, et al. Prognostic implications of echocardiographically determined left ventricular mass in the Framingham Heart Study. New Engl J Med. 1990;322:1561-1566.

9. Levy D, Labib SB, Anderson KM, et al. Determinants of sensitivity and specificity of electrocardiographic criteria for left ventricular hypertrophy. Circulation. 1990;81:815-820.

10. Rautaharju PM, Zhou SH, Calhoun C. Ethnic differences in ECG ampliyudes in north American white, black, and Hispanic men and women. Effect of obesity and age. J Electrocardiology. 1994;27:20-31.

11. Devereux RB, Aloson DR, Lutas EM, et al. Echocardiographic assessment of left ventricular hypertrophy: Comparison to necropsy findings. Am J Cardiol. 1986;57:450-458.

12. Sokolow M, Lyon TP. The ventricular complex in left ventricular hypertrophy as obtained by unipolar precordial and limb leads. Am Heart J. 1949;37: 161-186.

13. Devereux RB, Reichek N. Echocardiographic determination of left ventricular mass in man: anatomic validation of the method. Circulation. 1977;55: 613-618.

14. The Japanese Society of Hypertension. Guidelines for the management of hypertension 2019. JHS. 2019;13-46.

15. Berry RB, Albertario $C L$, Harding $S M$, et al. The AASM manual for the scoring of sleep and associated events: rules, terminology, and technical specification. J Clin Sleep Med. 2017;13:665-666.
16. Burger HC, Milaan JB. Heart vector and leads. Brit Heart J. 1946;8:157161.

17. Burger HC, Milaan JB. Heart vector and leads. Part II. Brit Heart J. 1947;9:154-160.

18. Burger HC, Milaan JB. Heart vector and leads. Part III. Geometrical representation. Brit Heart J. 1948;10:229-233.

19. Nagata Y. The influence of the inhomogeneities of electrical conductivity within the torso on the electrocardiogram as evaluated from the view point of the transfer impedance vector. Jpn Heart J. 1970;11:489-505.

20. Antman EM, Green LH, Grossman W. Physiologic determinants of the electrocardiographic diagnosis of left ventricular hypertrophy. Circulation 1979;60:386-396.

21. Dunn RA, Pipberger HV, Holt JH Jr, et al. Performance of conventional orthogonal and multiple-dipole electrocardiograms in estimating left ventricular muscle mass. Circulation. 1979;60:1350-1353.

22. Noda A, Yagi T, Yamada $\mathrm{H}$, et al. Electrocardiographic diagnosis of left ventricular hypertrophy improved by considering both QRS voltage and ST-T criteria. Jpn Circ J. 1994;58:698-706.

23. Noda A, Okada T, Yasuma, F, et al. Prognosis of the middle-aged and aged patients with obstructive sleep apnea syndrome. Psychiatry Clin. Neurosci. 1998; 52: 79-85.

24. Noda A, Nakata S, Koike Y, et al. Continuous positive airway pressure improves daytime baroreflex sensitivity and nitric oxide production in patients with moderate to severe obstructive sleep apnea syndrome. Hypertens Res. 2007;30:669-676.

25. Noda A, Hayano J, Ito N, et al. Very low frequency component of heart rate variability as a marker for therapeutic efficacy in patients with obstructive sleep apnea: Preliminary study. J Res Med Sci. 2019;24:84.

26. Nishizawa T, Cheng XW, Jin Z, et al. $\mathrm{Ca}(2+)$ channel blocker benidipine promotes coronary angiogenesis and reduces both left-ventricular diastolic stiffness and mortality in hypertensive rats. J Hypertens. 2010;28:1515-1526

27. Ichihara S, Noda A, Nagata K, et al. Pravastatin increases survival and suppresses an increase in myocardial matrix metalloproteinase activity in a rat model of heart failure. Cardiovasc Res. 2006;69:726-735. 